\title{
Intensity dependence of multiple orbital contributions and shape resonance in high-order harmonic generation of aligned $\mathbf{N}_{2}$ molecules
}

\author{
Cheng Jin, ${ }^{1}$ Julien B. Bertrand, ${ }^{2}$ R. R. Lucchese,${ }^{3}$ H. J. Wörner, ${ }^{4}$ Paul B. Corkum, ${ }^{2}$ D. M. Villeneuve, ${ }^{2}$ \\ Anh-Thu Le, ${ }^{1}$ and C. D. Lin ${ }^{1}$ \\ ${ }^{1}$ J. R. Macdonald Laboratory, Physics Department, Kansas State University, Manhattan, Kansas 66506-2604, USA \\ ${ }^{2}$ Joint Laboratory for Attosecond Science, National Research Council of Canada and University of Ottawa, 100 Sussex Drive, \\ Ottawa, Ontario K1A OR6, Canada \\ ${ }^{3}$ Department of Chemistry, Texas A\&M University, College Station, Texas 77843-3255, USA \\ ${ }^{4}$ Laboratorium für Physikalische Chemie, ETH Zürich, Wolfgang-Pauli-Strasse 10, 8093 Zürich, Switzerland
}

(Received 18 October 2011; published 9 January 2012)

\begin{abstract}
We report measurements and theoretical simulations of high-order harmonic generation (HHG) in aligned $\mathrm{N}_{2}$ molecules using a 1200-nm intense laser field when the generating pulse is perpendicular to the aligning one. With increasing laser intensity, the minimum in the HHG spectra first shifts its position and then disappears. Theoretical simulations including the macroscopic propagation effects in the medium reproduce these observations and the disappearance of the minimum is attributed to the additional contribution of HHG from inner orbitals. We also predict that the well-known shape resonance in the photoionization spectra of $\mathrm{N}_{2}$ should exist in the HHG spectra. It is most clearly seen when the generating laser is parallel to the aligning one and disappears gradually as the angle between the two lasers increases. No clear evidence of this shape resonance has been reported so far when using lasers with different wavelengths. Further experimentation is needed to draw conclusions.
\end{abstract}

DOI: 10.1103/PhysRevA.85.013405

PACS number(s): 33.80.Rv, 42.65.Ky, 31.70.Hq, 33.80.Eh

\section{INTRODUCTION}

Recently high-order harmonic generation (HHG) has been employed to probe the electronic structure of molecules on an ultrafast time scale [1-5]. After an electron is first removed from the molecule by the infrared (ir) laser, it may be driven back by the action of an intense laser field to recollide with the parent ion. HHG is a process where the returning electrons recombine with the molecular ion with the emission of highenergy photons. In fact, the last step- "recombination"-is the inverse of photoionization. Any spectral features in the photoionization cross section (PICS) would thus be embodied in the HHG spectra as well. Since molecules can be impulsively aligned by a laser field [6,7], the observation of HHG from aligned molecules further offers the opportunity for probing PICSs from aligned molecules that are not generally possible with synchrotron radiation experiments. In addition, the first step of HHG is a highly nonlinear tunneling ionization process which is very selective with respect to the ionization energy of the molecular orbital from which the electron is removed. Thus HHG in general is dominated by the recombination to the highest occupied molecular orbital (HOMO). In contrast, direct photoionization of a molecule is a linear process, where PICSs from the next or next few inner orbitals could be of comparable importance. For example, for $\mathrm{N}_{2}$, the HOMO is a $\sigma_{g}$ orbital. The next more tightly bound orbital, HOMO-1, is a $\pi_{u}$ orbital with an ionization energy $1.3 \mathrm{eV}$ higher. HOMO-2, is a $\sigma_{u}$ orbital and is $3.2 \mathrm{eV}$ more tightly bound than the HOMO. Calculations by Lucchese et al. [8] and experimental results $[9,10]$ show that for photon energy from the threshold at $15 \mathrm{eV}$ to about $40 \mathrm{eV}$, the PICS from HOMO-2 is negligible, but the HOMO-1 PICS is actually comparable with that of the HOMO. On the other hand, the HOMO has a shape resonance that peaks near the photon energy of $29 \mathrm{eV}$. These are predictions and measurements made on randomly oriented $\mathrm{N}_{2}$ molecules. How do these features depend on the alignment of molecules? Today the rich structure in PICSs from fixed-in-space molecules predicted by the theory remains mostly unexplored experimentally. Can high-harmonic spectra generated by laser pulses from aligned molecules provide new information that is not yet directly available from photoionization measurements? The answer is yes. Already experiments $[11,12]$ have shown the existence of a minimum in the HHG spectra from aligned molecules which has not been seen in photoionization experiment. Moreover, McFarland et al. [13] reported that HHG from HOMO-1 dominates over that from the HOMO when the molecules are perpendicularly aligned with respect to the laser polarization. Mairesse et al. [14] performed harmonic spectroscopy to characterize the attosecond dynamics of multielectron rearrangement during strong-field ionization.

Earlier attempts to extract structure information from HHG spectra were based on the two-center interference model [15] and the strong-field approximation (SFA) [16-18]. Subsequently, a quantitative rescattering (QRS) theory [19-21] was developed which established that HHG spectra from an isolated molecule can be expressed as the product of a returning electron wave packet with a photorecombination cross section (PRCS). The QRS asserts that the PRCS is independent of the laser parameters, including the wavelength, intensity, and pulse duration. The latter affect the returning electron wave packet only. In the meanwhile, Smirnova et al. [2] studied HHG from aligned $\mathrm{CO}_{2}$ molecules and emphasized the importance of hole dynamics with including multiple orbitals. Within the QRS theory, multiple orbitals can be easily incorporated into the theory and it was used by Le et al. [22] to explain the HHG data by McFarland et al. [13]. These theoretical studies were all based on single-molecule calculations. To compare with experimental measurements, however, the effect of the propagation of the driving laser field and the harmonics in the medium has to be considered. Until recently, the propagation 
effect has only been considered for atomic targets, but not for molecules [23-26]. Even for atoms, the propagation effect is considered mostly only within the SFA. Since experimental HHG spectra depend critically on laser parameters, focusing conditions, gas-jet pressure, and how the spectra are collected, these parameters have to be available in order to simulate the experimental spectra. When the experimental conditions are well specified, we have shown that direct comparison with experimental $\mathrm{HHG}$ spectra is now possible, for example, for $\mathrm{N}_{2}$ [23,24] and $\mathrm{CO}_{2}$ [25]. These comparisons are also becoming more interesting with the emergence of the use of midinfrared lasers to cover a broader range of photon energies.

In this combined theoretical and experimental article, we revisit the $\mathrm{HHG}$ spectra of $\mathrm{N}_{2}$ molecules that are aligned perpendicular to the laser polarization. This was previously studied by McFarland et al. [13] using 800-nm lasers near the cutoff region. Here we use 1200-nm lasers. We revisit the issue about the importance of the HOMO-1 contribution raised in Refs. [13,22,27]. We find that HOMO-1 does not contribute to the HHG spectra at low laser intensities, but becomes comparable to the HOMO at higher intensities. We also examine the shape resonance in the differential PICS and show how they would appear in the HHG spectra of aligned $\mathrm{N}_{2}$. The article is organized as follows. In Sec. II, information on the experimental setup is given. In Sec. III, we briefly summarize the theoretical methods used in the simulation. In Sec. IV, we show the comparison between experimental and theoretical HHG spectra, the differential PICS of the HOMO and HOMO-1, and the shape resonance in the HHG spectra of the HOMO for aligned $\mathrm{N}_{2}$. A short summary in Sec. V concludes this article.

\section{EXPERIMENTAL SETUP}

To perform the experiment, we use the output of a Ti:sapphire multipass laser system $(32 \mathrm{fs}, 800 \mathrm{~nm}, 50 \mathrm{~Hz}, 12 \mathrm{~mJ}$ per pulse), an optical setup to both split and recombine laser pulses, and a high-harmonic chamber, described previously [28], composed of a source chamber (pulsed valve, $250-\mu \mathrm{m}$ orifice) and an extreme ultraviolet (xuv) spectrometer.

First, we impulsively align molecules [6,7] with a stretched nonionizing pump pulse $\left(70 \pm 5 \mathrm{fs}, 800 \mathrm{~nm}, I_{\text {align }}=5 \times\right.$ $\left.10^{13} \mathrm{~W} / \mathrm{cm}^{2}\right)$. Second, we probe them with a delayed intense high-harmonic generation pulse (1200 nm: $40 \pm 5$ fs of variable intensity). The $1200-\mathrm{nm}$ pulses $(\sim 1 \mathrm{~mJ})$ come from a high-energy optical parametric amplifier (HE-TOPAS) pumped by $800-\mathrm{nm}$ light $(\sim 8 \mathrm{~mJ})$. In both arms of our Mach-Zehnder interferometer, the intensity is adjusted with neutral absorption-type density filters. Both linearly polarized pump and probe beams are focused $(f=50 \mathrm{~cm}) \sim 1 \mathrm{~mm}$ downstream in the gas jet expansion and $\sim 2 \mathrm{~mm}$ before the orifice to select only the so-called "short" trajectories [29].

We record high-harmonic spectra at maximal alignment at the half revival $(\sim 4.12 \mathrm{ps})$. Our experimental conditions $\left(P_{\text {back }}=2 \mathrm{~atm}, T_{\text {rot }}=30-40 \mathrm{~K}, I_{\text {align }}=5 \times 10^{13} \mathrm{~W} / \mathrm{cm}^{2}\right.$, and $\tau_{\text {align }}=70 \pm 5 \mathrm{fs}$ ) suggest we reach a maximum degree of alignment of $\left\langle\cos ^{2} \theta\right\rangle=0.60 \pm 0.05$ based on calculations [30] and supported by recent supersonic gas expansion studies under similar conditions [31]. We vary the angle $\alpha$ between the alignment axis and the probing field by rotating the alignment beam polarization; both beams are linearly polarized. This article concentrates on the case where $\alpha=90^{\circ}$.

\section{THEORETICAL METHODS}

The details of the theoretical methods used in the simulations have been described in Refs. [24,25]. Briefly, both the fundamental laser field and the xuv light are modified when they copropagate through a macroscopic medium. For some circumstances, such as low laser intensity, low gas pressure, and short gas medium, the effects of dispersion, Kerr nonlinearity, and plasmas defocusing on the fundamental laser field can be neglected [24,32]. Under these conditions the profile of the fundamental laser field in space (in the vacuum) can be expressed in an analytical form. If the ir laser is considered to be a Gaussian beam, its spatial and temporal dependence is given approximately in Ref. [32]. For the propagation of the harmonics in the medium, the dispersion and absorption effects, which are generally anisotropic in the case of aligned molecules, are not included when the pressure is low [24]. We only include the induced dipoles for the harmonics generated by the ir laser. The harmonics emitted from the gas medium propagate in the vacuum after the gas jet, and then they are detected by the spectrometer. A Hankel transformation [33] is applied to obtain the farfield harmonics using the harmonics at the exit of the gas medium.

The three-dimensional Maxwell's wave equation for the xuv light in the gas medium is [24,34-37]

$$
\nabla^{2} E_{h}^{\|}(r, z, t, \alpha)-\frac{1}{c} \frac{\partial^{2} E_{h}^{\|}(r, z, t, \alpha)}{\partial^{2} t}=\mu_{0} \frac{\partial^{2} P_{\mathrm{nl}}^{\|}(r, z, t, \alpha)}{\partial^{2} t} .
$$

Here $E_{h}^{\|}(r, z, t, \alpha)$ and $P_{\mathrm{nl}}^{\|}(r, z, t, \alpha)$ are the parallel components (with respect to the polarization direction of the generating laser) of the electric field of the xuv light and the nonlinear polarization caused by the ir laser, respectively. $\alpha$ is the pumpprobe angle, i.e., the angle between the aligning (pump) and the generating (probe) laser polarizations.

The nonlinear polarization term can be expressed as

$$
P_{\mathrm{nl}}^{\|}(r, z, t, \alpha)=\left[n_{0}-n_{e}(r, z, t, \alpha)\right] D^{\|, \text {tot }}(r, z, t, \alpha),
$$

where $n_{0}-n_{e}(r, z, t, \alpha)$ gives the density of the remaining neutral molecules and $D^{\|, \text {tot }}(t, \alpha)$ is the parallel component of the induced single-molecule dipole over a number of active electrons (including the effects from outermost and the inner orbitals). The QRS theory [19-21] is applied to calculate the induced dipole $D^{\| \text {,tot }}(t, \alpha)$ from each orbital. Within the QRS, the laser-induced dipole moment $D(\omega, \theta)$ for a fixed-in-space molecule (the molecular axis has an angle $\theta$ with respect to the laser polarization) is given by

$$
D^{\|}(\omega, \theta)=N(\theta)^{1 / 2} W(\omega) d^{\|}(\omega, \theta),
$$

where $N(\theta)$ is the alignment-dependent ionization probability, $W(\omega)$ is the recombining electron wave packet, and $d^{\|}(\omega, \theta)$ 
is the parallel component of the photorecombination (PR) transition dipole (complex in general).

For partially aligned molecules, we define an ionization weighted PR transition dipole moment by

$$
d^{\text {avg }}(\omega, \alpha)=\int_{0}^{\pi} N(\theta)^{1 / 2} d^{\|}(\omega, \theta) \rho(\theta, \alpha) \sin \theta d \theta,
$$

where $\rho(\theta, \alpha)$ is the alignment distribution in the probe-laser frame [38]. Such a definition can be made for each orbital from which the ionization occurs. The harmonic signal from each orbital, after propagation, can be expressed as $[23,24,32]$

$$
S_{h}(\omega, \alpha) \propto \omega^{4}|W(\omega)|^{2}\left|d^{\text {avg }}(\omega, \alpha)\right|^{2},
$$

where $W^{\prime}(\omega)$ is the "macroscopic wave packet" (MWP). If more than one molecular orbital contributes to the HHG spectra, then the transition dipole will be added up coherently. Note that in Eq. (3) the $N(\theta)$ for each molecular orbital is calculated using the molecular Ammosov-Delone-Krainov (MO-ADK) theory.

Based on the previous studies of $\mathrm{N}_{2}$ molecules, only the HOMO and HOMO-1 orbitals, which are $\sigma$ and $\pi$ orbitals, respectively, need to be included in the HHG calculation. We use either the terms HOMO and HOMO- 1 or $\sigma$ and $\pi$ orbitals interchangeably below.

\section{RESULTS AND DISCUSSION}

\section{A. Macroscopic HHG spectra: Experiment vs theory}

The HHG spectra for unaligned and aligned $\mathrm{N}_{2}$ and $\mathrm{CO}_{2}$ molecules using 800-nm and 1200-nm lasers have been reported recently [39]. Comparison with theoretically simulated spectra including macroscopic propagation has been carried out by Jin et al. [23-25]. In this article we present new measurements for aligned $\mathrm{N}_{2}$ molecules at a time delay corresponding to the maximal alignment while the HHG-generating laser is perpendicular to the aligning one. The degree of alignment is estimated to be $\left\langle\cos ^{2} \theta\right\rangle=0.60 \pm 0.05$.

Figure 1 shows the $\mathrm{HHG}$ spectra of aligned $\mathrm{N}_{2}$ generated by a 1200-nm laser which is perpendicular to the aligning laser. Experimentally, the laser duration (FWHM) is $\sim 44 \mathrm{fs}$, the beam waist at the focus is $\sim 40 \mu \mathrm{m}$, and the length of gas jet is $\sim 1 \mathrm{~mm}$, which is located $3 \mathrm{~mm}$ after the laser focus. A vertical slit with a width of $100 \mu \mathrm{m}$ is placed $24 \mathrm{~cm}$ after the gas jet. The intensities (in the center of the gas jet) used in the theory are adjusted to coincide with experimental HHG cutoff positions. In Figs. 1(a)-1(c), the laser intensities in theory (experiment) are $0.75(0.65), 0.9(1.1)$, and $1.1(1.3)$, in units of $10^{14} \mathrm{~W} / \mathrm{cm}^{2}$, respectively. We use the degree of alignment $\left\langle\cos ^{2} \theta\right\rangle=0.60$ and keep other parameters close to experimental ones in the simulation. The HHG spectra from the experiment and theory are normalized at the cutoff.

The main features in the spectra are the deep minima at 38.2 and $40.4 \mathrm{eV}$, at the two lower intensities in Figs. 1(a) and 1(b), respectively. The minimum disappears at the higher intensity in Fig. 1(c). To simulate the spectra at the two low intensities, we include the $\sigma$ orbital only. The simulation reproduces not only the correct shape of the spectra, but also the precise positions of the minima in the spectra in Figs. 1(a) and 1(b). For the higher intensity in Fig. 1(c), when we only included the $\sigma$ orbital
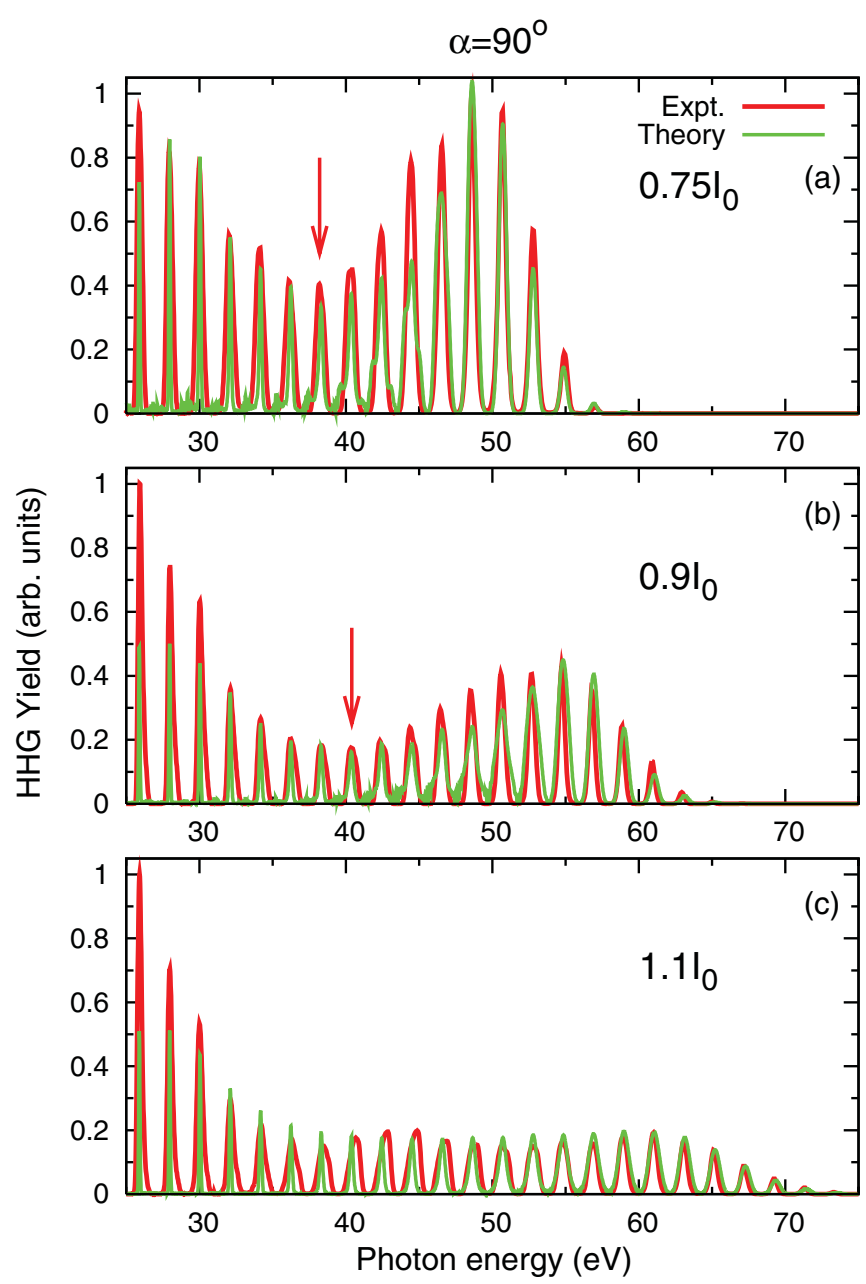

FIG. 1. (Color online) Comparison of experimental [red (dark gray) lines] and theoretical [green (light gray) lines] HHG spectra of aligned $\mathrm{N}_{2}$ in a 1200-nm generating laser where the pump-probe angle $\alpha=90^{\circ}$. Laser intensities in the simulations are indicated where $I_{0}=10^{14} \mathrm{~W} / \mathrm{cm}^{2}$. Degree of alignment is $\left\langle\cos ^{2} \theta\right\rangle=0.60$ by the aligning laser. See text for additional laser parameters. Only the $\sigma$ orbital is included in the simulations in panels (a) and (b), and both $\sigma$ and $\pi$ orbitals are included in the simulation in panel (c). Arrows indicate the positions of minima.

the theory could not reproduce the correct spectral shape. It also predicted a minimum in the spectrum which was not seen in the experiment [see Fig. 2(a)]. We then included both $\sigma$ and $\pi$ orbitals. A very good agreement between theory and experiment (correct shape and no minimum in the spectrum) in Fig. 1(c) is then achieved.

\section{B. Single HOMO orbital contribution at low laser intensity}

We now make a careful examination of the spectral features in Figs. 1(a) and 1(b). The deep minimum is related to the $\sigma$ orbital. This minimum has been observed in many experiments, either in unaligned or aligned $\mathrm{N}_{2}$ [39-42]. The minimum shifts only slightly when the laser intensity is changed. This behavior is similar to the well-known Cooper minimum in $\operatorname{Ar}[23,43,44]$. The same behavior with laser intensity has been observed by Wörner et al. [39] (see their 

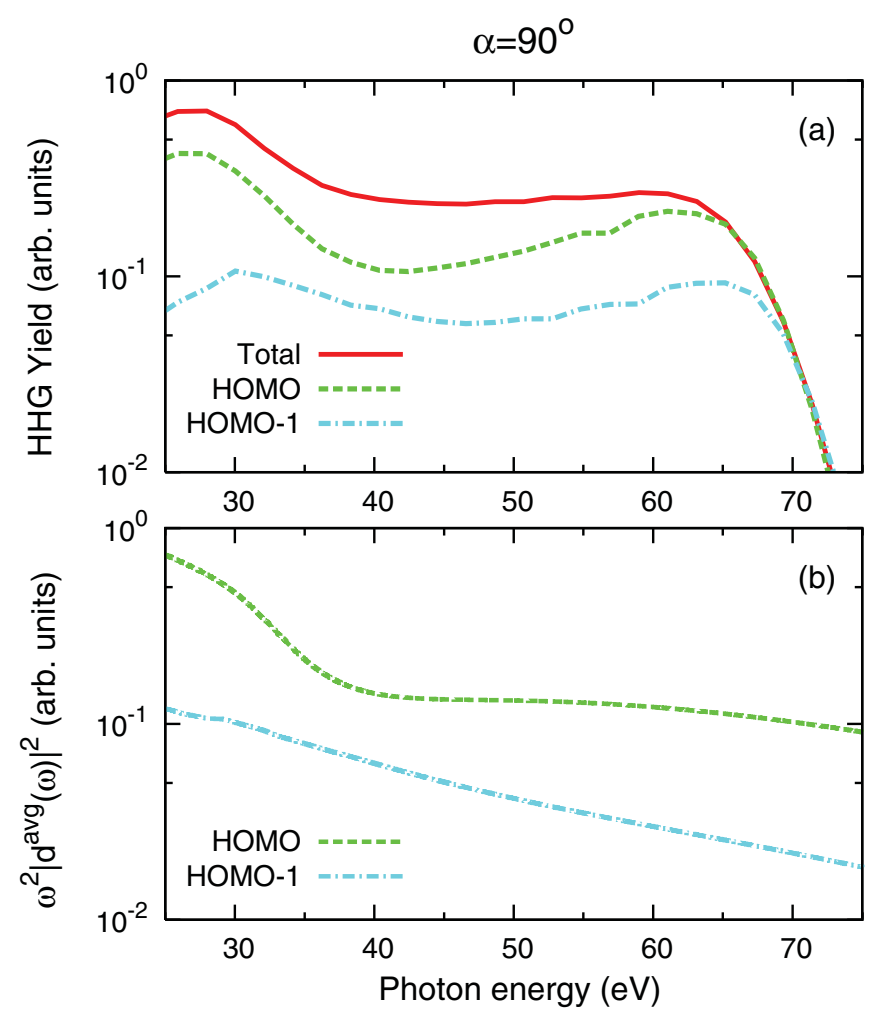

FIG. 2. (Color online) (a) Calculated macroscopic HHG spectra (envelope only) corresponding to Fig. 1(c). Total (HOMO and HOMO-1 together) spectra and individual HOMO and HOMO-1 spectra. (b) Averaged photorecombination transition dipoles (parallel component, the square of the magnitude multiplied by $\omega^{2}$ ) of the HOMO and HOMO-1 corresponding to panel (a). Laser intensity is $1.1 \times 10^{14} \mathrm{~W} / \mathrm{cm}^{2}$. Degree of alignment is $\left\langle\cos ^{2} \theta\right\rangle=0.60$ by the aligning laser. The pump-probe angle $\alpha=90^{\circ}$.

Fig. 1) and Farrell et al. [42] (see their Fig. 7) when $\alpha=0^{\circ}$. This can be understood as proposed in Ref. [23]. When only one molecular orbital is contributing to the HHG spectra, the harmonic yield is given in Eq. (5). In Fig. 2 we show that there is a fast dropoff in the averaged PR transition dipole (shown is the square of the magnitude multiplied by $\omega^{2}$ ) of the $\sigma$ orbital around $38 \mathrm{eV}$ [see Fig. 2(b)], which causes the pronounced minimum in the HHG spectra. For different laser intensities, the slope of the averaged PR transition dipole does not change, but the MWP $\left(\omega^{2}|W(\omega)|^{2}\right)$ increases monotonically with photon energy. Since the spatial distribution of the ir laser intensity is different when the peak intensity at the focus is increased, the wave packet is modified differently as it propagates through the medium (see Fig. 3(d) in Ref. [23]). This makes the minimum in the HHG spectra shift slightly with laser intensity. Using only one molecular orbital, our theoretical simulation can reproduce the position of the minimum close to the experimental one.

\section{Multiple orbital contributions (HOMO and HOMO-1) at higher laser intensity}

We next analyze the spectral features in Fig. 1(c). In Fig. 2(a), the envelopes of the HHG spectra in Fig. 1(c) from the two individual molecular orbitals, and from the total one, obtained after macroscopic propagation in the gas medium are shown. Meanwhile, the averaged PR transition dipoles (the square of the magnitude multiplied by $\omega^{2}$, degeneracy is not included) of the two orbitals are shown in Fig. 2(b).

In Fig. 2(a), the $\sigma$ orbital alone shows a deep minimum, similar to Figs. 1(a) and 1(b). Since the $\pi$ orbital shows comparable contributions over the whole spectral region, the interference between $\sigma$ and $\pi$ orbitals washes out the minimum in the spectra. From Eq. (4), the relative contribution between the two orbitals can be adjusted by the alignment-dependent ionization probabilities $N(\theta)$ and the alignment distribution $\rho(\theta, \alpha)$. For lower intensities, the relative $N(\theta)$ of the $\pi$ orbital is small, so only the HOMO orbital contributes to the spectra. As the intensity increases, both orbitals contribute and the two amplitudes interfere resulting in a drastic change of the spectra.

As shown in Eq. (5), the macroscopic HHG spectra from individual molecular orbitals can be considered as a product of a MWP and an averaged PR transition dipole, based on the QRS theory. Since the ionization potential of the $\sigma$ orbital $(15.6 \mathrm{eV})$ differs from that of the $\pi$ orbital $(16.9 \mathrm{eV})$ only by $1.3 \mathrm{eV}$, the MWPs of the two orbitals are almost the same under the same ir laser, so the relative contribution between $\sigma$ and $\pi$ orbitals to the total HHG spectra is mostly determined by the averaged PR transition dipoles. In Figs. 1(a) and 1(b), the magnitude of $N(\theta)$ for the $\sigma$ orbital is much larger than the one for the $\pi$ orbital, thus making the corresponding averaged PR transition dipole also larger. At the higher intensity of Fig. 1(c), the averaged transition dipoles between the two orbitals become comparable. Thus by increasing the laser intensity, the total HHG can evolve from single-orbital to multiple-orbital phenomena. Note that the MO-ADK theory $[45,46]$ is used to calculate $N(\theta)$. There are also other models in the literature $[22,47,48]$ for calculating the ionization rates. Using different ionization rates and different alignment distributions may change the theoretical predictions. We find that the ionization rates obtained from MO-ADK theory are very close to the recent model calculations used by Petretti et al. [48] where they solved the time-dependent Schrödinger equation at a laser intensity of $1.5 \times 10^{14} \mathrm{~W} / \mathrm{cm}^{2}$.

The contribution of the $\pi$ orbital to the HHG of $\mathrm{N}_{2}$ when the pump-probe angle $\alpha=90^{\circ}$ has been studied previously $[13,27]$ using $800-\mathrm{nm}$ pulses at high intensities of around $2.0 \times 10^{14} \mathrm{~W} / \mathrm{cm}^{2}$. In this case, HOMO-1 was found to be much more pronounced in the cutoff region. Note that the QRS theory has been applied to interpret the results in Ref. [13] with the ionization probability obtained from SFA [22] without including the propagation effect. (Note that at the alignment angle of $90^{\circ}$, the ratio of ionization rate between the HOMO and HOMO- 1 is about 2 from the SFA in comparison with about 5 from the MO-ADK theory $[45,46]$.) In this study, a $1200-n m$ laser generates a broad photon energy range even with a low laser intensity and the $\pi$ orbital contributes not only in the cutoff region but also in the plateau.

In the future, measurements similar to the present one but with a full range of pump-probe angles may provide a way to determine the relative ionization probabilities of the two orbitals. The pump-probe angle $\alpha=90^{\circ}$ is much closer to the alignment angle of $90^{\circ}$ since the "volume element" $\sin \theta d \theta$ in 
the angular integration peaks at $90^{\circ}$. To probe the $\mathrm{PR}$ transition dipole of the HOMO orbital, HHG spectra taken at low laser intensity with long-wavelength lasers are preferable to avoid multiple orbital contributions. This would make the retrieval of the structure of the target easier. If one wishes to study the $\pi$ orbital, then a higher laser intensity and better alignment will enhance its contribution to the HHG [13,22].

\section{Photoionization amplitudes and phases of $\mathrm{N}_{2}$ from $\mathrm{HOMO}$ and HOMO-1 orbitals}

The most basic information on photoionization of a fixedin-space molecule is the parallel and perpendicular transition dipole amplitudes in the body-fixed frame of the molecule. They appear in the differential PICS in the body-fixed frame as $[8,49]$

$$
\frac{d^{2} \sigma}{d \Omega_{\hat{k}} d \Omega_{\hat{n}}}=\frac{4 \pi^{2} \omega k}{c}\left|\left\langle\Psi_{i}|\vec{r} \cdot \hat{n}| \Psi_{f, \vec{k}}^{(-)}\right\rangle\right|^{2},
$$

where $\hat{n}$ is the polarization direction of the light, $\vec{k}$ the momentum of the photoelectron, and $\omega$ the photon energy. We only focus on the case of $\hat{n} \| \vec{k}$ since they are related to the parallel polarized HHG spectra measured from aligned molecules.

In Fig. 3 we show the differential PICSs (in units of $\mathrm{Mb}$ ) and the corresponding phases of the $\sigma$ and $\pi$ orbitals of $\mathrm{N}_{2}$ using the well-established photoionization theory $[8,49]$ for photon energy from 20 to $80 \mathrm{eV}$. These data have been shown previously for selected photon energies against the alignment angles for the HOMO (see Fig. 5 of Ref. [21]) and HOMO-1 (see Fig. 2 of Ref. [22]). The observed HHG minima shown in Fig. 1 above for $\alpha=90^{\circ}$ and in Fig. 2 of Ref. [23] for $\alpha=0^{\circ}$ and for randomly distributed $\mathrm{N}_{2}$ molecules can all be understood as due to the rapid change of cross section near
$40 \mathrm{eV}$ at alignment angles close to either $0^{\circ}$ or $90^{\circ}$. Clearly the precise position of the minimum will depend on the degree of alignment. For HOMO-1, Fig. 3 shows that the cross section generally peaks at large alignment angles. Thus interference of HHG spectra from the $\sigma$ and $\pi$ orbitals is only observed close to $\alpha=90^{\circ}$. Note that in Fig. 3 the PICSs for the two orbitals are shown on the same scale. Except for the HOMO shape resonance near $30 \mathrm{eV}$ that is be discussed next, the PICS from HOMO-1 is comparable with that from HOMO over the photon energies covered. This result has been confirmed by the measured electron spectra in photoionization experiments. In $\mathrm{HHG}$, the HOMO contribution is always dominant for the randomly distributed $\mathrm{N}_{2}$. HOMO- 1 can become more important only at large alignment angles and higher laser intensities, as discussed earlier.

\section{E. Shape resonance in $\mathrm{HHG}$ of aligned $\mathbf{N}_{\mathbf{2}}$}

Resonances are very common in photoionization spectra. Most of them are due to the so-called Feshbach resonances, which in general are quite narrow and thus can only be observed using high-resolution spectroscopy. However, broad Feshbach resonances and shape resonances can be observed since they have widths from fractions to a few electron volts. In $\mathrm{HHG}$, resonances have been explored in experiment [50] and theory [51-54] recently for atomic targets. But shape resonances are rare for common atomic targets. The resonance feature observed in $\mathrm{Xe}$ [50] is due to the intershell coupling with the well-known shape resonance that occurs in the $4 d$ shell. On the other hand, shape resonances are very common in molecules. For $\mathrm{N}_{2}$, there is a pronounced shape resonance in the HOMO channel near $30 \mathrm{eV}$. This is due to the $3 \sigma_{g} \rightarrow k \sigma_{u}$ channel, and for small alignment angles only. We can see a decrease of the phase shift by $\pi$ for this resonance from 20 to $40 \mathrm{eV}$ in Fig. 3(b). There are no other known shape resonances
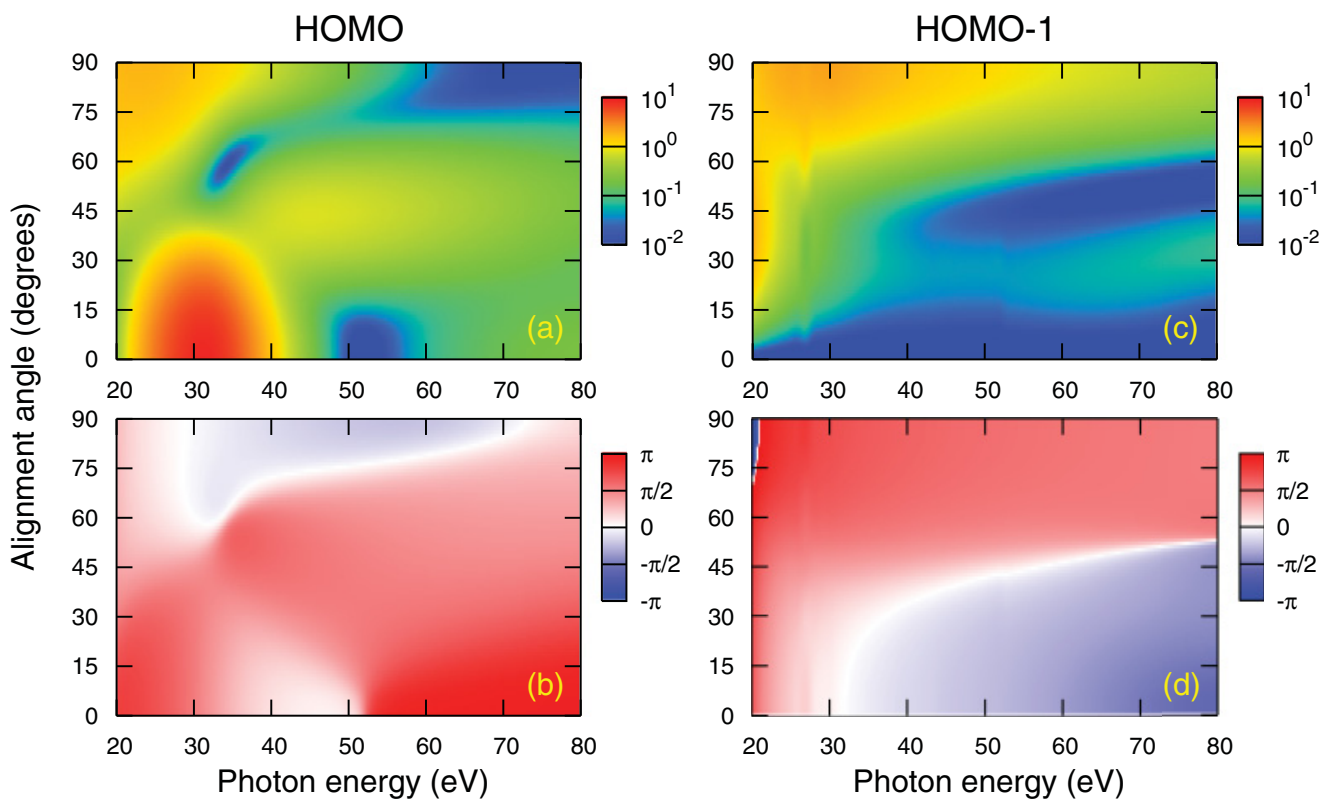

FIG. 3. (Color online) Calculated differential photoionization cross sections [(a) and (c)] and phases [(b) and (d)] of the HOMO and HOMO- 1 for $\mathrm{N}_{2}$ in terms of the alignment angles, respectively. The shape resonance in the HOMO shows up around $30 \mathrm{eV}$ for small alignment angles only. Only the parallel component to the polarization direction of the laser is shown. 


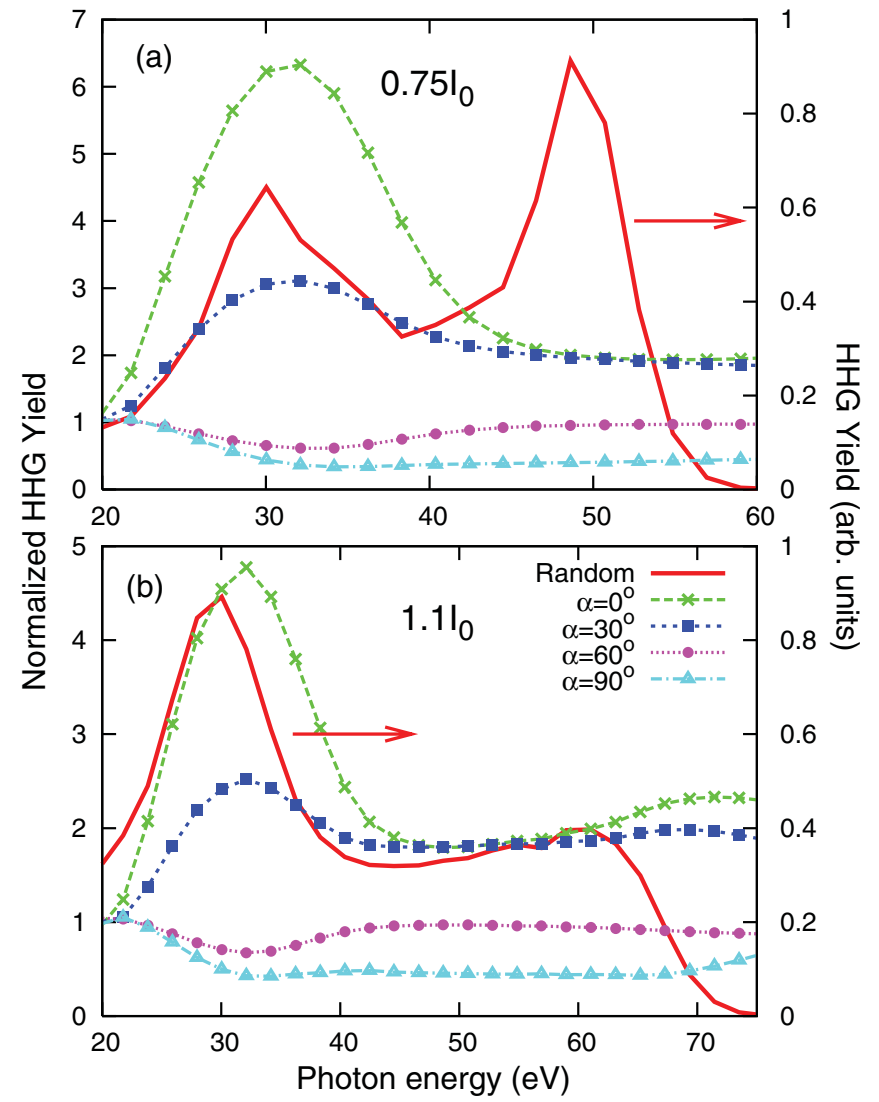

FIG. 4. (Color online) Calculated macroscopic HHG spectra (envelope only) of unaligned $\mathrm{N}_{2}$ and the normalized HHG yields of aligned $\mathrm{N}_{2}$ with respect to the unaligned ones as a function of the photon energy at the selected pump-probe angle $\alpha$. Laser intensities are indicated where $I_{0}=10^{14} \mathrm{~W} / \mathrm{cm}^{2}$. Degree of alignment is $\left\langle\cos ^{2} \theta\right\rangle=0.60$.

in the covered energy region. Clearly this shape resonance is best observed by selecting ionization from the $\sigma$ orbital only, by using low laser intensity, and for molecules that are aligned nearly parallel to the polarization axis of the probing laser.

In Fig. 4, we first show the calculated HHG spectra (envelope only, normalized) of randomly distributed $\mathrm{N}_{2}$ at two laser intensities. For randomly distributed $\mathrm{N}_{2}$ molecules, it is known that the $\sigma$ orbital is the dominant contributor to the HHG spectra. The peak around $30 \mathrm{eV}$ in the HHG spectra is due to the shape resonance in the PICS of the $\sigma$ orbital at small alignment angles. In Fig. 4, we also show the normalized yield at selected alignment angles with respect to the randomly distributed one. We take the degree of alignment $\left\langle\cos ^{2} \theta\right\rangle=0.60$. The intensity of each odd harmonic $q$ is obtained by integrating over harmonics $q-1$ to $q+1$. For low laser intensity, the shape resonance is very pronounced at $\alpha=0^{\circ}$. It decreases as the pump-probe angle is increased, showing that the shape resonance is present only at small aligning angles. For higher laser intensities, the same behavior is seen even though the $\pi$ orbital is also contributing at large pump-probe angles. We should comment that absorption is not included in the present simulation and it will suppress the shape resonance if the gas pressure is high.
Comparison of this prediction with existing experimental data is far from conclusive. Torres et al. [40] have shown high-harmonics data for aligned $\mathrm{N}_{2}$ using a 1300-nm laser with an intensity of $1.3 \times 10^{14} \mathrm{~W} / \mathrm{cm}^{2}$ (see their Fig. 4). The general trend of their data is very close to our prediction, but they used a higher intensity and a higher degree of alignment $\left(\left\langle\cos ^{2} \theta\right\rangle=0.66\right)$, and thus the HOMO- 1 channel may contribute to the signal at larger alignment angles. Using 800-nm lasers, Lee et al. [27] reported the HHG ratios of aligned vs unaligned $\mathrm{N}_{2}$ at the selected alignment angles. They did not present data near the resonance region and they used a high intensity of $2.5 \times 10^{14} \mathrm{~W} / \mathrm{cm}^{2}$ [see their Fig. 1(c)]. Our calculation does not reproduce their measured ratios. They also reported that the normalized HHG yields for $\alpha=0^{\circ}$ and $90^{\circ}$ cross at about harmonic order 39 . This is confirmed in our calculation (not shown). The very recent measurement by Kato et al. [55] did not extend below $30 \mathrm{eV}$ either. There are other measurements $[28,42,56]$ using $\operatorname{Ar}$ or $\alpha=0^{\circ}$ as a reference. Direct comparison with these data is difficult. Thus, it remains to be seen if the shape resonance in $\mathrm{N}_{2}$ can be seen in the HHG spectra as predicted here. We comment that absorption was not included in our propagation simulation. Absorption may modify the prediction if the gas pressure is too high. Experiments dedicated to address this issue would be of interest.

\section{CONCLUSIONS AND OUTLOOK}

In this article, we have reported experimental HHG spectra of aligned $\mathrm{N}_{2}$ at a time delay of the maximal alignment using a 1200-nm laser when the pump-probe angle is set at $90^{\circ}$. The minimum of the HHG spectra appears at about 38-40 eV at two low laser intensities. It disappears at a higher laser intensity. We have carried out theoretical simulations to understand these results and concluded that the minima in the HHG are associated with the properties of the transition dipole moments for photoionization from the HOMO. At higher intensity, the contribution from HOMO-1 becomes important and interference between the two contributions washes out the minimum.

We have also examined the possibility of observing the well-known shape resonance in the photoionization of $\mathrm{N}_{2}$ in the HHG spectra. While the normalized HHG yield (with respect to randomly distributed molecules) shows clear enhancement at small alignment angles and shape resonance may have been seen in the $1300-n m$ data, no evidence of shape resonance has been observed in the HHG spectra from 800-nm lasers. Further experiments dedicated to resolve this issue will be of great interest.

\section{ACKNOWLEDGMENTS}

This work was supported in part by the Chemical Sciences, Geosciences and Biosciences Division, Office of Basic Energy Sciences, Office of Science, US Department of Energy. J.B.B. was also supported by CIPI, NSERC, and AFOSR. C.J. and C.D.L. thank Gae Hwang Lee and Chang Hee Nam for communicating their experimental results. 
[1] J. Itatani, J. Levesque, D. Zeidler, H. Niikura, H. Pépin, J. C. Kieffer, P. B. Corkum, and D. M. Villeneuve, Nature (London) 432, 867 (2004).

[2] O. Smirnova, Y. Mairesse, S. Patchkovskii, N. Dudovich, D. Villeneuve, P. Corkum, and M. Yu. Ivanov, Nature (London) 460, 972 (2009).

[3] H. J. Wörner, J. B. Bertrand, D. V. Kartashov, P. B. Corkum, and D. M. Villeneuve, Nature (London) 466, 604 (2010).

[4] S. Haessler, J. Caillat, and P. Salières, J. Phys. B 44, 203001 (2011).

[5] A. T. Le, R. R. Lucchese, M. T. Lee, and C. D. Lin, Phys. Rev. Lett. 102, 203001 (2009).

[6] H. Stapelfeldt and T. Seideman, Rev. Mod. Phys. 75, 543 (2003).

[7] F. Rosca-Pruna and M. J. J. Vrakking, Phys. Rev. Lett. 87, 153902 (2001).

[8] R. R. Lucchese, G. Raseev, and V. McKoy, Phys. Rev. A 25, 2572 (1982).

[9] E. W. Plummer, T. Gustafsson, W. Gudat, and D. E. Eastman, Phys. Rev. A 15, 2339 (1977).

[10] A. Hamnett, W. Stoll, and C. E. Brion, J. Electron Spectrosc. Relat. Phenom. 8, 367 (1976).

[11] C. Vozzi et al., Phys. Rev. Lett. 95, 153902 (2005).

[12] R. Torres et al., Phys. Rev. A 81, 051802 (2010).

[13] B. K. McFarland, J. P. Farrell, P. H. Bucksbaum, and M. Gühr, Science 322, 1232 (2008).

[14] Y. Mairesse et al., Phys. Rev. Lett. 104, 213601 (2010).

[15] M. Lein, N. Hay, R. Velotta, J. P. Marangos, and P. L. Knight, Phys. Rev. Lett. 88, 183903 (2002).

[16] M. Lewenstein, Ph. Balcou, M. Yu. Ivanov, A. L'Huillier, and P. B. Corkum, Phys. Rev. A 49, 2117 (1994).

[17] X. X. Zhou, X. M. Tong, Z. X. Zhao, and C. D. Lin, Phys. Rev. A 71, 061801 (2005).

[18] X. X. Zhou, X. M. Tong, Z. X. Zhao, and C. D. Lin, Phys. Rev. A 72, 033412 (2005).

[19] C. D. Lin, A. T. Le, Z. Chen, T. Morishita, and R. R. Lucchese, J. Phys. B 43, 122001 (2010).

[20] T. Morishita, A. T. Le, Z. Chen, and C. D. Lin, Phys. Rev. Lett. 100, 013903 (2008).

[21] A. T. Le, R. R. Lucchese, S. Tonzani, T. Morishita, and C. D. Lin, Phys. Rev. A 80, 013401 (2009).

[22] A. T. Le, R. R. Lucchese, and C. D. Lin, J. Phys. B 42, 211001 (2009).

[23] C. Jin, H. J. Wörner, V. Tosa, A. T. Le, J. B. Bertrand, R. R. Lucchese, P. B. Corkum, D. M. Villeneuve, and C. D. Lin, J. Phys. B 44, 095601 (2011).

[24] C. Jin, A. T. Le, and C. D. Lin, Phys. Rev. A 83, 023411 (2011).

[25] C. Jin, A. T. Le, and C. D. Lin, Phys. Rev. A 83, 053409 (2011).

[26] S. F. Zhao, C. Jin, R. R. Lucchese, A. T. Le, and C. D. Lin, Phys. Rev. A 83, 033409 (2011).

[27] G. H. Lee, I. J. Kim, S. B. Park, T. K. Kim, Y. S. Lee, and C. H. Nam, J. Phys. B 43, 205602 (2010).

[28] Y. Mairesse, J. Levesque, N. Dudovich, P. B. Corkum, and D. M. Villeneuve, J. Mod. Opt. 55, 2591 (2008).
[29] P. Saliéres, A. L'Huillier, and M. Lewenstein, Phys. Rev. Lett. 74, 3776 (1995).

[30] Y. Mairesse, N. Dudovich, D. Zeidler, M. Spanner, D. M. Villeneuve, and P. B. Corkum, J. Phys. B 43, 065401 (2010).

[31] K. Yoshii, G. Miyaji, and K. Miyazaki, Opt. Lett. 24, 1651 (2009).

[32] C. Jin, A. T. Le, and C. D. Lin, Phys. Rev. A 79, 053413 (2009).

[33] A. E. Siegman, Lasers (University Science, Mill Valley, CA, 1986).

[34] M. B. Gaarde, J. L. Tate, and K. J. Schafer, J. Phys. B 41, 132001 (2008).

[35] E. Priori, G. Cerullo, M. Nisoli, S. Stagira, S. De Silvestri, P. Villoresi, L. Poletto, P. Ceccherini, C. Altucci, R. Bruzzese, and C. de Lisio, Phys. Rev. A 61, 063801 (2000).

[36] V. Tosa, H. T. Kim, I. J. Kim, and C. H. Nam, Phys. Rev. A 71, 063807 (2005)

[37] M. Geissler, G. Tempea, A. Scrinzi, M. Schnürer, F. Krausz, and T. Brabec, Phys. Rev. Lett. 83, 2930 (1999).

[38] M. Lein et al., J. Mod. Opt. 52, 465 (2005).

[39] H. J. Wörner, J. B. Bertrand, P. Hockett, P. B. Corkum, and D. M. Villeneuve, Phys. Rev. Lett. 104, 233904 (2010).

[40] R. Torres et al., Opt. Express 18, 3174 (2010).

[41] B. K. McFarland, J. P. Farrell, P. H. Bucksbaum, and M. Gühr, Phys. Rev. A 80, 033412 (2009).

[42] J. P. Farrell, B. K. McFarland, M. Gühr, and P. H. Bucksbaum, Chem. Phys. 366, 15 (2009).

[43] H. J. Wörner, H. Niikura, J. B. Bertrand, P. B. Corkum, and D. M. Villeneuve, Phys. Rev. Lett. 102, 103901 (2009).

[44] S. Minemoto, T. Umegaki, Y. Oguchi, T. Morishita, A. T. Le, S. Watanabe, and H. Sakai, Phys. Rev. A 78, 061402 (2008).

[45] X. M. Tong, Z. X. Zhao, and C. D. Lin, Phys. Rev. A 66, 033402 (2002).

[46] S. F. Zhao, C. Jin, A. T. Le, T. F. Jiang, and C. D. Lin, Phys. Rev. A 81, 033423 (2010).

[47] D. A. Telnov and Shih-I. Chu, Phys. Rev. A 79, 041401 (2009).

[48] S. Petretti, Y. V. Vanne, A. Saenz, A. Castro, and P. Decleva, Phys. Rev. Lett. 104, 223001 (2010).

[49] C. Jin, A. T. Le, S. F. Zhao, R. R. Lucchese, and C. D. Lin, Phys. Rev. A 81, 033421 (2010).

[50] A. D. Shiner, B. E. Schmidt, C. Trallero-Herrero, H. J. Wörner, S. Patchkovskii, P. B. Corkum, J.-C. Kieffer, F. Légaré, and D. M. Villeneuve, Nat. Phys. 7, 464 (2011).

[51] V. Strelkov, Phys. Rev. Lett. 104, 123901 (2010).

[52] M. Tudorovskaya and M. Lein, Phys. Rev. A 84, 013430 (2011).

[53] C. Jin, A. T. Le, C. A. Trallero-Herrero, and C. D. Lin, Phys. Rev. A 84, 043411 (2011).

[54] M. V. Frolov, N. L. Manakov, A. A. Silaev, N. V. Vvedenskii, and A. F. Starace, Phys. Rev. A 83, 021405 (2011).

[55] K. Kato, S. Minemoto, and H. Sakai, Phys. Rev. A 84, 021403 (2011).

[56] S. Haessler et al., Nat. Phys. 6, 200 (2010). 